\title{
A simple spectral condition implying separability for states of bipartite quantum systems
}

\author{
M E Gabach Clément and G A Raggio \\ FaMAF, Universidad Nacional de Córdoba, X5000 Córdoba, Argentina \\ E-mail: raggio@famaf.unc.edu.ar
}

\begin{abstract}
We give a simple spectral condition in terms of the ordered eigenvalues of the state of a bipartite quantum system which is sufficient for separability.
\end{abstract}

PACS numbers: 03.65.Ud, 03.67.-a

We consider quantum systems where the underlying Hilbert space $\mathcal{H}$ is the tensor product of two finite dimensional Hilbert spaces. A state of such a system is identified with a density operator, and is said to be separable if it can be written as a convex sum of pure product states of the system; that is to say vector states where the vectors are product vectors. The separable states form a convex subset of the states of the system.

For the simplest bipartite composite system we have the following result

Theorem 1 If the eigenvalues $\lambda_{1} \geq \lambda_{2} \geq \lambda_{3} \geq \lambda_{4}$ of the two qubit state $\rho$ satisfy $3 \lambda_{1}+\sqrt{2} \lambda_{2}+(3-\sqrt{2}) \lambda_{3} \leq 2$, then $\rho$ is separable.

The states satisfying the inequality have spectra in the simplex spanned by the spectra (always written taking into account multiplicities and nonincreasingly) $(1 / 2,1 / 6,1 / 6,1 / 6),((2+\sqrt{2}) / 8,(2+\sqrt{2}) / 8,(2-\sqrt{2}) / 8,(2-\sqrt{2}) / 8),(1 / 3,1 / 3,1 / 3,0)$ and $(1 / 4,1 / 4,1 / 4,1 / 4)$.

The method used to prove the above result also gives a different proof of the following result given in [1] (Theorem 3)

Theorem 2 If the eigenvalues $\lambda_{1} \geq \lambda_{2} \geq \cdots \geq \lambda_{d}$ of the state $\rho$ of a bipartite quantum system of dimension $d$ satisfy $3 \lambda_{d}+(d-1) \lambda_{d-1} \geq 1$, then $\rho$ is separable.

Both results provide simple spectral criteria ensuring separability. In the case of two qubits $(d=4)$ Theorem 2 is much weaker than Theorem 1.

Before proceeding to the proofs, we compare theorem 1 with other available results of the same nature-that is: conditions on the spectrum implying separability of the state. Given a state $\rho$ of a $d$-dimensional bipartite quantum system, we let $\operatorname{spec}(\rho)=$ $\left(\rho_{1}, \rho_{2}, \cdots, \rho_{d}\right)$ denote the vector of repeated eigenvalues of $\rho$ enumerated so that $\rho_{1} \geq \rho_{2} \geq \cdots \geq \rho_{d}$. In the two qubit case, let $\Sigma=\left\{\left(\lambda_{1}, \lambda_{2}, \lambda_{3}, \lambda_{4}\right): \lambda_{1} \geq \lambda_{2} \geq \lambda_{3} \geq\right.$ $\left.\lambda_{4} \geq 0, \lambda_{1}+\lambda_{2}+\lambda_{3}+\lambda_{4}=1\right\}$ be all possible state spectra. Theorem 1 asserts that if $\operatorname{spec}(\rho)$ lies in $\mathcal{A}:=\left\{\lambda \in \Sigma: 3 \lambda_{1}+\sqrt{2} \lambda_{2}+(3-\sqrt{2}) \lambda_{3} \leq 2\right\}$, then $\rho$ is separable. One of the first and most useful results of this nature is that of [2]: If $\operatorname{tr}\left(\rho^{2}\right) \leq 1 / 3$ then $\rho$ is separable. In terms of spectra this is: $\operatorname{spec}(\rho) \in \mathcal{B}:=\left\{\lambda \in \Sigma: \lambda_{1}^{2}+\lambda_{2}^{2}+\lambda_{3}^{2}+\lambda_{4}^{2} \leq 1 / 3\right\}$ 
implies $\rho$ is separable. Although $\mathcal{A} \cap \mathcal{B}$ is quite large, $\mathcal{A} \neq \mathcal{B}$, and the conditions defining $\mathcal{A}$ and $\mathcal{B}$ capture different (convex) sets of separable states. To see this, observe that $\lambda \equiv((2+\sqrt{2}) / 8,(2+\sqrt{2}) / 8,(2-\sqrt{2}) / 8,(2-\sqrt{2}) / 8) \in \mathcal{A}$ but $\lambda_{1}^{2}+\lambda_{2}^{2}+\lambda_{3}^{2}+\lambda_{4}^{2}=$ $3 / 8>1 / 3$. Moreover, $(\sqrt{2} / 3)(3 / 4,1 / 4,0,0)+(1-(\sqrt{2} / 3))(1 / 4,1 / 4,1 / 4,1 / 4)$ lies in $\mathcal{B}$ but not in $\mathcal{A}$. The determination of "maximally entangled" states of two qubits by Verstraete, Audenaert and De Moor, 3], has the following as a consequencef: let $\mathcal{C}:=\left\{\lambda \in \Sigma: \lambda_{1}-\lambda_{3}-2 \sqrt{\lambda_{2} \lambda_{4}} \leq 0\right\}$; then $\rho$ is separable if $\operatorname{spec}(\rho) \in \mathcal{C}$. The inequality $\sqrt{(t a+(1-t) b)(t c+(1-t) d)} \geq t \sqrt{a c}+(1-t) \sqrt{b d}$ valid for $0 \leq t \leq 1$ and $a, b, c, d \geq 0$ shows inmediately that $\mathcal{C}$ is convex. By the results of [3], one has $\mathcal{B} \subset \mathcal{C} \S$ One verifies that the four vertices of $\mathcal{A}$ given after the statement of theorem 1 lie in $\mathcal{C}$ so that $\mathcal{A} \subset \mathcal{C}$ because $\mathcal{A}$ is the convex hull of its four vertices.

The proof of the two stated results uses certain tools developped in [1] which we briefly present. Consider the maximally mixed state $\tau=1 / \operatorname{dim}(\mathcal{H})$, then $\tau$ factorizes over the two factors of $\mathcal{H}$ so that $\tau$ is a separable state. Consider the segment with endpoints $\rho$ and $\tau: \rho_{t}=t \cdot \rho+(1-t) \cdot \tau, 0 \leq t \leq 1$. The modulus of separability $\ell$ [1], measures how far you can go towards $\rho$ beginning at $\tau$ until you lose separability: $\ell(\rho)=\sup \left\{t: \rho_{t}\right.$ is separable $\}$. The quantity $(1 / \ell)-1$ was studied by Vidal and Tarrach 4 as the "random robustness of entanglement". It can be shown (4, 1]) that the supremum is a maximum; that $\rho_{t}$ is separable iff $t \leq \ell(\rho)$; that $\ell(\rho)>0$; and that $1 / \ell$ is a convex map on the states: for states $\rho, \phi$ and $0 \leq s \leq 1$,

$$
\ell(s . \rho+(1-s) \cdot \phi) \geq\left(\frac{s}{\ell(\rho)}+\frac{1-s}{\ell(\phi)}\right)^{-1} .
$$

The other ingredient is the so called gap-representation of a state introduced in [1]. Let $\operatorname{spec}(\rho)=\left(\lambda_{1}, \lambda_{2}, \cdots, \lambda_{d}\right)$ and $\rho=\sum_{j=1}^{d} \lambda_{j} \cdot \rho_{j}$ be a spectral decomposition of $\rho$ where the $\rho_{j}$ are pairwise orthogonal pure vector states. Define $\mu_{j}=j\left(\lambda_{j}-\lambda_{j+1}\right)$, $j=1,2, \cdots, d-1 ; \widehat{\rho}_{j}=j^{-1} \sum_{m=1}^{j} \rho_{m}, j=1,2, \cdots, d$. Notice that $\sum_{j=1}^{d-1} \mu_{j}=1-d \lambda_{d} ;$ that $\widehat{\rho}_{d}=\tau ;$ and that

$$
\operatorname{spec}\left(\widehat{\rho}_{j}\right)=(\underbrace{1 / j, 1 / j, \cdots, 1 / j}_{j}, 0, \cdots, 0) \equiv e^{(j)} .
$$

So $\widehat{\rho}_{1}$ is pure. Then a gap-representation of $\rho$ is $\rho=\sum_{j=1}^{d-1} \mu_{j} \cdot \widehat{\rho}_{j}+d \lambda_{d} \cdot \tau$. Noticing that $\lambda_{d}=1 / d$ iff $\rho=\tau$, we assume that this is not the case and write

$$
\rho=\left(1-d \lambda_{d}\right) \cdot \omega+d \lambda_{d} \cdot \tau, \quad \omega=\sum_{j=1}^{d-1} \frac{\mu_{j}}{1-d \lambda_{d}} \cdot \widehat{\rho}_{j} .
$$

By the results mentioned, $\rho$ is separable iff

$$
\left(1-d \lambda_{d}\right) \leq \ell(\omega) .
$$

$\ddagger$ We thank H. Vogts and K. Życzkowski for bringing [3] to our attention.

$\S$ A direct proof goes as follows. It suffices to show that if $\lambda \in \mathcal{B}$ with $\lambda_{1}^{2}+\lambda_{2}^{2}+\lambda_{3}^{2}+\lambda_{4}^{2}=1 / 3$ then $\lambda \in \mathcal{C}$. Now putting $\lambda_{4}=1-\lambda_{1}-\lambda_{2}-\lambda_{3}$ in the previous identity, we obtain

$$
\lambda_{1}^{2}+\lambda_{2}^{2}+\lambda_{3}^{2}-\left(\lambda_{1}+\lambda_{2}+\lambda_{3}\right)+\left(\lambda_{1} \lambda_{2}+\lambda_{1} \lambda_{3}+\lambda_{2} \lambda_{3}\right)=-1 / 3 .
$$

Then, $\left(\lambda_{1}-\lambda_{3}\right)^{2}-4 \lambda_{2} \lambda_{4}=\lambda_{1}^{2}+\lambda_{3}^{2}-6 \lambda_{1} \lambda_{3}-4 \lambda_{2}+4 \lambda_{2}^{2}+4\left(\lambda_{1} \lambda_{2}+\lambda_{1} \lambda_{3}+\lambda_{2} \lambda_{3}\right)$. Using the displayed identity to eliminate the summand $4\left(\lambda_{1} \lambda_{2}+\lambda_{1} \lambda_{3}+\lambda_{2} \lambda_{3}\right)$, we obtain $\left(\lambda_{1}-\lambda_{3}\right)^{2}-4 \lambda_{2} \lambda_{4}=$ $-3\left(\lambda_{1}+\lambda_{3}-2 / 3\right)^{2} \leq 0$, and this proves that $\lambda \in \mathcal{C}$. 
Applying (11) to the state $\omega$ in its gap representation, we have

$$
\ell(\omega) \geq\left(\sum_{j=1}^{d-1} \frac{\mu_{j}}{\left(1-d \lambda_{d}\right) \ell\left(\widehat{\rho}_{j}\right)}\right)^{-1}=\left(1-d \lambda_{d}\right)\left(\sum_{j=1}^{d-1} \frac{\mu_{j}}{\ell\left(\widehat{\rho}_{j}\right)}\right)^{-1} ;
$$

thus (2) is satisfied (and thus $\rho$ is separable) if $\sum_{j=1}^{d-1} \mu_{j} / \ell\left(\widehat{\rho}_{j}\right) \leq 1$. We can replace the $\ell\left(\widehat{\rho}_{j}\right)$ by lower bounds.

Proposition If $\ell\left(\widehat{\rho}_{j}\right) \geq p_{j} \geq 0$ for $j=1,2, \cdots, d-1$ and $\sum_{j=1}^{d-1} \mu_{j} / p_{j} \leq 1$ then $\rho$ is separable.

The prime reason for introducing the gap-representation is that not only the last summand $\tau$ but also the second last $\widehat{\rho}_{d-1}$ are separable. This follows from a result of Gurvits and Barnum [5] : If $\operatorname{tr}\left(\phi^{2}\right) \leq 1 /(d-1)$ for a bipartite composite system of dimension $d$ then $\phi$ is separable. Now indeed $\operatorname{tr}\left(\widehat{\rho}_{d-1}^{2}\right)=1 /(d-1)$.

The least possible modulus of separability has been computed by Vidal and Tarrach 4]: $\inf \{\ell(\phi): \phi$ a state $\}=2 /(2+d)$; the infimum is assumed at a pure state. To prove theorem 2, put $p_{1}=p_{2}=\cdots=p_{d-2}=2 /(2+d)$ and $p_{d-1}=1$ in the proposition.

Turning to theorem 1, consider the numbers $\widehat{\ell}_{j}:=\inf \left\{\ell(\phi): \operatorname{spec}(\phi)=e^{(j)}\right\}$, which give the minimal moduli of separability for the states spanning all possible gaprepresentations. Replacing $p_{j}$ by $\widehat{l}_{j}$ in the Proposition gives us a general inequality providing a sufficient condition for separability. No general information is available for the $\vec{l}_{j}$ except the calculation of [6] for two qubits where $\widehat{l}_{1}=1 / 3, \widehat{l}_{2}=1 / \sqrt{2}$, and $\widehat{l}_{3}=1$. From this and the proposition one gets theorem 1 . Since $\widehat{l}_{1}=1 / 3$, and $\widehat{l}_{3}=1$ follow from the results quoted above, we only give the calculation of $\widehat{l}_{2}$ in the appendix.

\section{Appendix A. Calculation of $\ell$ for a two qubit state with} $\operatorname{spec}=(1 / 2,1 / 2,0,0)$

[6] gives a direct calculation of $\widehat{\ell}_{1}, \widehat{\ell}_{2}$ and $\widehat{\ell}_{3}$ using the Wootters Criterion [7]. Recall that if $\rho$ is a state of a two qubit system, the Wootters operator $W$ asssociated to it is $W=\left(\sqrt{\rho}\left(\sigma_{y} \otimes \sigma_{y}\right) \bar{\rho}\left(\sigma_{y} \otimes \sigma_{y}\right) \sqrt{\rho}\right)^{1 / 2}$. Here all operators are taken as matrices with respect to a product orthonormal basis.

$$
\sigma_{y}=\left(\begin{array}{cc}
0 & -i \\
i & 0
\end{array}\right)
$$

and $\bar{\rho}$ is the complex conjugate of $\rho$ taken with respect to the basis which is real. The Wootters Criterion is: $\rho$ is separable if and only if the (repeated) eigenvalues $w_{1} \geq w_{2} \geq w_{3} \geq w_{4}$ of $W$ satisfy $w_{1} \leq w_{2}+w_{3}+w_{4}$.

We will calculate the modulus of separability for any state $\rho$ for which $\operatorname{spec}(\rho)=$ $(1 / 2,1 / 2,0,0)$ by calculating the spectrum of the Wootters operator associated to $\rho_{t}=t \rho+(1-t) \tau, 0 \leq t \leq 1$. The spectrum of $\rho_{t}$ consists of two double eigenvalues $\alpha=(1+t) / 4$ and $\beta=(1-t) / 4$ (which coincide for $t=0$ where $\rho_{0}=\tau$ ). In order not to overload the notation we consider a density operator $A$ with $\operatorname{spec}(A)=(\alpha, \alpha, \beta, \beta)$ where $\alpha+\beta=1 / 2$, and $\alpha \geq \beta \geq 0$; thus $1 / 4 \leq \alpha \leq 1 / 2$. The spectral decomposition of $A$ reads $A=\alpha P+\beta P^{\perp}$, where $P$ is an orthoprojection of rank 2 and $P^{\perp}=\mathbf{1}-P$ is its orthocomplement, another orthoprojection of rank 2 . It follows that $\left(\sigma_{y} \otimes \sigma_{y}\right) \bar{A}\left(\sigma_{y} \otimes \sigma_{y}\right)=\alpha Q+\beta Q^{\perp}$, where $Q=\left(\sigma_{y} \otimes \sigma_{y}\right) \bar{P}\left(\sigma_{y} \otimes \sigma_{y}\right)$ is an orthoprojection 
of rank 2 and $Q^{\perp}=\mathbf{1}-Q$. Using this one obtains for the square of the Wootters operator associated to $A$ the formula $W^{2}=\beta^{2} \mathbf{1}+\beta(\alpha-\beta)(P+Q)+(\alpha-\beta)(\sqrt{\alpha \beta}-$ $\beta)(P Q+Q P)+\left(\alpha^{2}-\beta^{2}-2 \sqrt{\alpha \beta}(\alpha-\beta)\right) P Q P$. Now since $P, P^{\perp}, Q$ and $Q^{\perp}$ are orthoprojections of rank 2 in a four dimensional Hilbert space, we have three mutually exclusive alternatives for the subspaces $U$ and $V$ spanned by $P$ and $Q$ respectively: (1) $U \cap V=\{0\}$ which happens when and only when $Q=\mathbf{1}-P$ which is equivalent to $\operatorname{tr}(P Q)=0 ;(2) \operatorname{dim}(U \cap V)=2$ which happens when and only when $Q=P$ which is equivalent to $\operatorname{tr}(P Q)=2$; and (3) $\operatorname{dim}(U \cap V)=1$ which happens when and only when there are unit vectors $\psi, \phi$ and $\chi$ in the four dimensional Hilbert space which satisfy $\langle\psi, \phi\rangle=\langle\psi, \chi\rangle=0$ and $|\langle\chi, \phi\rangle|<1$ such that $P=|\psi\rangle\langle\psi|+| \phi\rangle\langle\phi|$ and $Q=|\psi\rangle\langle\psi|+| \chi\rangle\langle\chi|$. One has $\operatorname{tr}(P Q)=1+|\langle\chi, \phi\rangle|^{2}$. This alternative is equivalent to $\operatorname{tr}(P Q) \in[1,2)$.

The three alternatives are distinguished by the value of $\operatorname{tr}(P Q)$. For convenience we introduce the following characteristic geometric parameter $\xi=\operatorname{tr}(P Q)-1$, which will determine the modulus of separability completely. We now distinguish the three possibilities.

(1) which occurs iff $\xi=-1$. Here $P Q=0$ allows one to compute $W^{2}=\alpha \beta \mathbf{1}$. The Wootters Criterion is satisfied and the associated state is separable.

(2) which occurs iff $\xi=1$. Here $P=Q$ allows one to calculate directly $W=A$, and the Wootters Criterion is just $\alpha \leq 1 / 2$ so the associated state is separable.

(3) which occurs iff $0 \leq \xi<1$. We may assume that

$$
\psi=\left(\begin{array}{l}
1 \\
0 \\
0 \\
0
\end{array}\right), \phi=\left(\begin{array}{l}
0 \\
1 \\
0 \\
0
\end{array}\right), \chi=\left(\begin{array}{c}
0 \\
\sqrt{\xi} \\
\eta_{1} \\
\eta_{2}
\end{array}\right), \eta=\left(\begin{array}{c}
\eta_{1} \\
\eta_{2}
\end{array}\right),
$$

where $\eta \neq 0$ because $\|\eta\|^{2}=\|\chi\|^{2}-\xi=1-\xi>0$. We now partition $\mathbb{C}^{4}=\mathbb{C} \oplus \mathbb{C} \oplus \mathbb{C}^{2}$, and doing the necessary matrix multiplications we get, from our previous formula for $W^{2}$,

$$
W^{2}=\left(\begin{array}{ccc}
\alpha^{2} & 0 & \langle 0| \\
0 & \alpha \beta+\alpha(\alpha-\beta) \xi & (\alpha-\beta) \sqrt{\xi \alpha \beta}\langle\eta| \\
|0\rangle & (\alpha-\beta) \sqrt{\xi \alpha \beta}|\eta\rangle & \beta^{2} \mathbf{1}_{2}+\beta(\alpha-\beta)|\eta\rangle\langle\eta|
\end{array}\right) .
$$

It is now clear that $\alpha^{2}$ is an eigenvalue of $W^{2}$. The eigenvalue condition for an eigenvalue $\zeta$ to the eigenvector $x \oplus \mu$ for the lower right $3 \times 3$ block on $\mathbb{C} \oplus \mathbb{C}^{2}$ is

$$
\begin{aligned}
& (\zeta-\alpha \beta-\alpha(\alpha-\beta) \xi) x=(\alpha-\beta) \sqrt{\xi \alpha \beta}\langle\eta, \mu\rangle \\
& \left(\zeta-\beta^{2}\right) \mu=(\alpha-\beta)(\sqrt{\xi \alpha \beta} x+\beta\langle\eta, \mu\rangle) \eta .
\end{aligned}
$$

Putting $x=0$ and taking as we may $\mu \neq 0$ orthogonal to $\eta$, A.1 is satisfied and (A.2) reduces to $\left(\zeta-\beta^{2}\right) \mu=0$, thus $\beta^{2}$ is an eigenvalue of $W^{2}$. We are now left with the problem of finding eigenvectors orthogonal to those already found. They are of the form $x \oplus c \eta$ with $x, c \in \mathbb{C}$. Inserting such eigenvectors into (A.1) and (A.2), the discussion of the solutions is tedious but straightforward. One obtains the two missing eigenvalues of $W^{2}$ to be $\zeta_{ \pm}(\alpha, \xi)=\frac{\alpha}{2}(1-2 \alpha)+\frac{\xi}{8}(4 \alpha-1)^{2} \pm$ $\frac{4 \alpha-1}{4} \sqrt{2 \xi \alpha(1-2 \alpha)+\xi^{2}(2 \alpha-1 / 2)^{2}}$. Having the four eigenvalues of $A$ we must decide which is the largest. We have $\alpha \geq \beta$ by assumption, and clearly $\zeta_{+}(\alpha, \xi) \geq \zeta_{-}(\alpha, \xi)$. Moreover, $\xi \mapsto \zeta_{+}(\alpha, \xi)$ is increasing and $\zeta_{+}(\alpha, 1)=\alpha^{2}$. Thus, $\alpha$ is the largest 
eigenvalue of $W$ and the Wootters Criterion reads: $\alpha \leq \beta+\sqrt{\zeta_{+}(\alpha, \xi)}+\sqrt{\zeta_{-}(\alpha, \xi)}$. Manipulation of this inequality shows that it is equivalent to $\alpha \leq(1+(1 / \sqrt{2-\xi})) / 4$.

Recalling that $\alpha=(1+t) / 4$, we arrive at: If the two qubit state $\rho$ has $\operatorname{spec}(\rho)=(1 / 2,1 / 2,0,0)$ then

$$
\ell(\rho)= \begin{cases}1 & , \quad \text { if } Q=\mathbf{1}-P \\ \frac{1}{\sqrt{3-\operatorname{tr}(P Q)}} \quad, \quad \text { otherwise }\end{cases}
$$

where $P$ is the spectral orthoprojection to the eigenvalue $1 / 2$ and $Q=\left(\sigma_{y} \otimes \sigma_{y}\right) \bar{P}\left(\sigma_{y} \otimes\right.$ $\left.\sigma_{y}\right)$. Since $\operatorname{tr}(P Q) \in[1,2]$ when $Q \neq \mathbf{1}-P$, we obtain $\widehat{\ell}_{2}=\inf \{\ell(\rho): \operatorname{spec}(\rho)=$ $(1 / 2,1 / 2,0,0)\}=1 / \sqrt{2}$.

\section{References}

[1] Raggio G A 2006 Spectral conditions on the state of a composite quantum system implying its separability J. Phys. A 39617

[2] Życzkowski K, Horodecki P, Sanpera A and Lewenstein M 1998 Volume of the set of separable states. Phys. Rev. A $\mathbf{5 8} 883$.

[3] Verstraete F, Audenaert K and De Moor B 2001 Maximally entangled mixed states of two qubits Phys. Rev. A 64012316.

[4] Vidal G and Tarrach R 1999 Robustness of entanglement Phys. Rev. A 59141

[5] Gurvits L and Barnum H 2003 Separable balls around the maximally mixed multipartite quantum state Phys. Rev. A $\mathbf{6 8} 042312$

[6] Gabach Clément M E 2005 Entrelazamiento en sistemas cuánticos compuestos. Trabajo Especial de Licenciatura en Física, FaMAF, Diciembre 2005. Unpublished.

[7] Wootters W K 1998 Entanglement of formation of an arbitrary state of two qubits Phys. Rev. Lett. 802245 